\title{
Characterizing planetesimal belts through the study of debris dust
}

\author{
Amaya Moro-Martín ${ }^{1,2}$ \\ ${ }^{1}$ Departamento de Astrofísica, CAB (CSIC-INTA), Instituto Nacional de Técnica \\ Aeroespacial, Torrejón de Ardoz, 28850, Madrid, Spain \\ email: amaya@cab.inta-csic.es \\ ${ }^{2}$ Department of Astrophysical Sciences, Princeton University, Peyton Hall, Ivy Lane, \\ Princeton, NJ 08544, USA
}

\begin{abstract}
Main sequence stars are commonly surrounded by disks of dust. From lifetime arguments, it is inferred that the dust particles are not primordial but originate from the collision of planetesimals, similar to the asteroids, comets and KBOs in our Solar system. The presence of these debris disks around stars with a wide range of masses, luminosities, and metallicities, with and without binary companions, is evidence that planetesimal formation is a robust process that can take place under a wide range of conditions. Debris disks can help us learn about the formation, evolution and diversity of planetary systems.
\end{abstract}

Keywords. interplanetary medium, Kuiper Belt, circumstellar matter, stars: evolution, planetary systems

\section{Why we care about debris disks}

Circumstellar disks play a fundamental role in the formation of stars and planets. The accretion of mass onto the forming star is regulated by mass and angular momentum transfer mechanisms within the disk. With time, the mass reservoir of the cloud gets depleted and the gas-rich disk begins to dissipate in a time scale of about 6 Myr. The formation of gas giant planets needs to happen before this gas-rich disk dissipates, while the formation of terrestrial planets and massive planets beyond the ice line is not limited by the presence of gas in the disk and can continue for approximately $100 \mathrm{Myr}$; a critical step in this process is the formation of planetesimals. Observations with Spitzer show that there is evidence that at least $15 \%$ of mature stars (10 Myr-10 Gyr) of a wide range of masses $\left(0.5-3 \mathrm{M}_{\text {Sun }}\right.$ ) harbor planetesimal belts with sizes of $10 \mathrm{~s}-100 \mathrm{~s}$ AU. This evidence comes from the presence of an infrared emission in excess of that expected from the stellar photosphere, thought to arise from a circumstellar dust disk. The reason why these dust disks are evidence of the presence of planetesimals is because the lifetime of the dust grains is of the order of $0.01-1 \mathrm{Myr}$, much shorter than the age of the star $(>10 \mathrm{Myr})$; therefore, the origin of these dust grains cannot be primordial, i.e. from the cloud of gas and dust where the star was born, but must be the result of on-going dust production generated by planetesimals, like the asteroids, comets and Kuiper Belt Objects (KBOs) in our Solar system; this is why we refer to these dust disks as debris disks. Debris disks are therefore evidence of the formation of planetesimals around other stars. The goal of this presentation is to describe how debris disks can shed light on the formation, evolution an diversity of planetary systems, helping us place our Solar system into context. 


\section{The Solar system debris disk}

The Sun also harbors a disk of dust produced in the inner and outer Solar system by the asteroids, comets and KBOs. Scattered light and thermal emission observations of the inner part of the Solar system debris disk (known as the zodiacal cloud) can help determine the properties of the dust particles, revealing non-spherical, rapidly-rotating, irregular or fluffy aggregates, $10-100 \mu \mathrm{m}$ in size, composed of a mixture of silicates and organic material with a low albedo; there is also a population of smaller $1 \mu \mathrm{m}$-sized grains made of crystalline olivine and hydrous silicate that accounts for a weak silicate emission feature at $10 \mu \mathrm{m}$ (Reach et al. 2003). The thermal emission from these dust particles dominates the night sky between $5-500 \mu \mathrm{m}$, with a fractional luminosity of $f=L_{\text {dust }} / L_{\odot} \sim 10^{-8}-10^{-7}$ (Dermott et al. 2002), more than two orders of magnitude fainter than the extra-solar debris disks observed with Spitzer. Regarding the spatial distribution, the thermal emission from the zodiacal cloud shows long, narrow arcs that coincide with the perihelion passage of some short-period comets, and broader dust bands at low ecliptic latitudes, thought to originate from the disruption events that gave rise to the asteroidal families Themis, Koronis and Eos (Sykes \& Greenberg 1986). The thermal emission also shows a ring-like structure that results from the trapping of dust particles in the exterior mean motion resonances (MMRs) with the Earth, with a 10\% number density enhancement on the Earth's wake that results from the resonance geometry (Dermott et al. 1994). Secular perturbations with the planets are thought to account for the offset of the zodiacal cloud center with respect to the Sun, the inclination of the cloud with respect to the ecliptic and the cloud warp.

Nesvorny et al. (2010) argued that the splitting of Jupiter family comets accounts for $85 \%$ of the dust in the inner Solar system. The dust production rate of comets is difficult to estimate because the cometary activity is not steady: sublimation drives the cometary activity in the inner Solar system, however, there are also isolated flare-ups that can produce dust at large heliocentric distances. Cometary dust particles have been studied in situ in the case of comets Halley, Tempel 1 and Wild 2. Dust particles have also been detected in situ at different heliocentric distances by the spacecrafts HEOS (1 AU), Hitten (1 AU), Helios (0.3-1AU), Galileo (0.7-5 AU), Pioneer 8 and 9 (0.75-1.08 AU), Ulysses (1.3-2.3 AU), Cassini and Pioneer 10 and 11 (see review by Grün et al. 2001). The current dust production rate in the inner Solar system is of the order of $10^{4}$ $\mathrm{kg} / \mathrm{s}$; the relative contribution of the different sources is still under debate and has likely changed with time. Pioneer 10 and 11 detected dust out to 18 AU and 13 AU, respectively (Humes 1980), and dynamical models indicate and that the Kuiper belt (KB) was likely the source of the dust detected beyond 10 AU (Landgraf et al. 2002). Voyager detected dust in the 30-60 AU Kuiper Belt region, with an estimated number density of $n \sim$ $2 \times 10^{-8} \mathrm{~m}^{-3}$ (Gurnett et al. 1997 ) that would correspond to a fractional luminosity of $f=L_{\text {dust }} / L_{\text {star }} \sim 4 \times 10^{-7}$ (Jewitt \& Luu 2000). The dust production rate estimates in the outer Solar system are in the rage $(0.2-5) \times 10^{4} \mathrm{~kg} / \mathrm{s}$ (from Voyager and Pioneer data, respectively; Jewitt \& Luu 2000; Landgraf et al. 2002).

The dust production rate in the Solar system has changed significantly with time. It is thought that the Solar system was significantly more dusty in the past because the asteroid belt $(\mathrm{AB})$ and the $\mathrm{KB}$ were more densely populated; with time, it became progressively less dusty as the planetesimal belts eroded away by mutual planetesimal collisions, leading to a $1 / t$ decay in the dust thermal emission. It is expected that this decay was punctuated by large spikes that are due to large collisions happening stochastically (examples of stochastic events in the recent history of the Solar system are the fragmentation of the asteroids giving rise to the asteroidal families and the dust bands). 
A major change in the dust production rate is expected to have occurred when the Solar system was $\sim 600$ Myr old, at the time of the Late Heavy Bombardment (LHB), thought to be caused by the orbital migration of the giant planets that produced a resonance sweeping of the $\mathrm{AB}$ that made the asteroids orbits unstable, causing a large scale ejection of bodies into planet-crossing orbits (explaining the observed cratering record Strom et al. 2005); the orbital migration of the planets also caused a major depletion of the KB as Neptune migrated outward (Gomes et al. 2005). After the LHB, there must have been a sharp decrease in the dust production rate due to the drastic depletion of planetesimal (Booth et al. 2009).

\section{Extra-solar debris disks: lessons from Spitzer}

Spitzer carried out extensive debris disks surveys. Taking advantage of the unprecedented sensitivity of the Spitzer/MIPS instrument, hundreds of debris disks were identified, allowing to characterize the frequency and properties of debris disks around stars of different spectral types, ages and environment. These are some of the main lessons learned.

\subsection{Debris disk frequency}

A survey of 328 solar-type FGK stars (30 Myr-3 Gyr) found that the frequency of $24 \mu \mathrm{m}$ excess (tracing dust around $3-5 \mathrm{AU}$ ) is $14.7 \%$ at $<300 \mathrm{Myr}$ and $2 \%$ at $>300 \mathrm{Myr}$, while at $70 \mu \mathrm{m}$ (tracing dust around 28-75 AU), the excess rates are $6-10 \%$ and are fairly independent of age (Meyer et al. 2008; Hillenbrand et al. 2008; Carpenter et al. 2009). Debris disks are more common around A-type stars than around FGK stars: a survey of 160 A-type stars showed that $32 \%$ and $\geqslant 33 \%$ of stars show excess emission at the 3- $\sigma$ confidence level at $24 \mu \mathrm{m}$ at $70 \mu \mathrm{m}$, respectively ( $\mathrm{Su}$ et al. 2006). On the other hand, debris disks are significantly less common around old M-type stars (Gautier et al. 2007), but this may be an observational bias because the peak emission of these colder disks would be at $\lambda>70 \mu \mathrm{m}$, i.e. beyond the wavelength where Spitzer/MIPS was most sensitive. On-going debris disks surveys with Herschel are doubling the number of disk detection rates made by Spitzer and, in fact, most of the new detected debris disks are found around cold late-type stars (Eiroa et al. in preparation, see proceeding in this volume). There is also evidence of the presence of planetesimals around white dwarfs: some of these evolved stars show infrared excesses and high levels of pollutants (elements other than the expected pure $\mathrm{H}$ and $\mathrm{He}$ ), thought to arise from tidally disrupted planetesimals (Jura 2006). The presence of planetesimals around stars with a very wide range of spectral types, from M-type to the progenitors of white dwarfs - with several orders of magnitude difference in stellar luminosities - implies that planetesimal formation is a robust process that can take place under a wide range of conditions.

\subsection{Debris disk fractional luminosities}

Due to the limited sensitivity of the Spitzer debris disks surveys, the detected fractional luminosities are generally $f=L_{d u s t} / L_{*} \gtrsim 10^{-5}$; this is larger than those inferred for the Solar system's debris disk today : $f \sim 10^{-8}-10^{-7}$ for the inner Solar system and the estimated $f \sim 10^{-7}-10^{-6}$ for the outer Solar system. Assuming a gaussian distribution of debris disk luminosities and extrapolating from Spitzer observations (that show a steep increase in the number of detections with decreasing $f$ ), Bryden et al. (2006) concluded the fractional luminosity of an average debris disk around a solar-type stars could be between $0.1-10 \times$ that of the Solar system debris disk. In other words, the observations are consistent with debris disks at the Solar system level being common (but too faint to 
be detected by Spitzer). On-going debris disks surveys with Herschel, sensitive to fainter and colder disks, are revisiting the frequency of disk detections.

\subsection{Debris disk evolution}

The study of the frequency and properties of debris disks around stars of different ages can shed light on the evolution of debris disks with time (see review by Wyatt 2008). Collisional models predict that the steady erosion of planetesimals will naturally lead to a decrease in the dust production rate; this slow decay will be punctuated by short-term episodes of increased activity triggered by large collisional events that can make the disk look an order of magnitude brighter. These models agree broadly with the observations derived from the Spitzer surveys. It is found that the frequency and fractional luminosities of debris disks around FGK stars with ages in the range 0.01-1 Gyr declines in a timescale of 100-400 Myr, but there is no clear evidence of a decline in the 1-10 Gyr age range (Trilling et al. 2008). This indicates that different physical processes might be dominating the evolution of the dust around the younger and the older systems. A possible scenario is that, at young ages, stochastic dust production due to individual collisions is more prominent, while at older ages, the steady grinding down of planetesimals dominates. The relative importance of these two processes is still under discussion. The Spizer surveys also showed that the evolution of dust around both A-type and FGK stars proceeds differently in the inner and outer regions, with the warmer dust (dominating the emission at $24 \mu \mathrm{m}$ ) declines faster than the colder dust (seen at $70 \mu \mathrm{m}$ ). This indicates that the clearing of the disk in the inner regions is more efficient, as would be expected from the shorter dynamical timescales. Regarding the issue of steady state vs. stochastic dust production, some systems show evidence that transient events dominate the dust production. This is the case of HD 69830, a star that shows no excess emission at $70 \mu \mathrm{m}$, but shows strong excess at $24 \mu \mathrm{m}$, with prominent spectral features in the Spizter/IRS wavelength range that are indicative of the presence of large quantities of small warm grains (Beichman et al. 2005). Because these small grains have very short lifetimes, it is inferred that the level of dust production is too high to be sustained for the age of the system (because the planetesimals would have not survived the inferred erosion rate), concluding that it is a transient event (Wyatt et al. 2007). HD 69830 is particularly interesting because it harbors three Neptune-like planets inside 1 AU (Lovis et al. 2006) and the best fit to the dust spectra is that of a disrupted, highly processed, low carbon P- or D-type asteroid (very common in the inner $\mathrm{AB}$ - Lisse et al. 2007), located near the 2:1 and 5:2 mean motion resonaces of the outermost planet; this raises the question whether the increased level of dust production is the result of gravitational perturbations by the planets.

As we mentioned above, there is evidence that the migration of the giant planets in the early Solar system had an important effect on the evolution of its debris disk: the drastic planetesimal clearing that resulted would have been associated with a sharp decreased in the dust production rate (Booth et al. 2009). Because the presence of hot Jupiters and multiple planets locked in resonances are evidence that planet migration has taken place in some planetary systems, a natural question to ask is whether debris disks observations show evidence of drastic planetesimal clearing events: a statistical study by Booth et al. (2009) concluded that less than $12 \%$ of solar-type star suffered LHB-type of events, but this is a preliminary result because Spitzer surveys were limited in sensitivity, so this issue needs to be revisited in the future with deeper surveys.

\subsection{Debris disk structure and inferred planetesimal location}

The few dozen debris disks that have been spatially resolved so far show a rich diversity of structural features, including narrow to wide belts, clumpy rings, sharp inner 
edges, brightness asymmetries, offsets of the dust disk center with respect to the central star, warps of the disk plane and spirals. Because some of these features have also been observed in the Solar system debris disk, where they are thought to be caused by the gravitational perturbations from the planets, a natural question to ask is whether the asymmetries observed in the extra-solar debris disks reveal the presence of unseen planetary companions: clumpy rings have been explained as dust and/or dust-producing planetesimals trapped in MMRs with a planet; warps can be the result of secular perturbations of a planet in an orbit inclined with respect to the planetesimal/dust disk; and spirals, offsets and brightness asymmetries might also be the result of secular perturbations, in this case of an eccentric planet that forces an eccentricity on the planetesimals and the dust. Because these structural features depend on the mass and orbit of the planet, this opens the possibility of using the study of the dust disk structure as a planet detection technique. The planets recently found in the Fomalhaut and $\beta$-Pic systems, predicted to exist to account for the structure observed in both disks, illustrate this idea (see proceedings by Kalas et al. and Lagrange et al. in this volume).

Even though most of the debris disks observed with Spitzer are spatially unresolved, limited information regarding the dust location can be extracted from the analysis of the disk spectral energy distribution (SED). A Spitzer survey of 328 FGK stars at 24 and $70 \mu \mathrm{m}$ found that about $2 / 3$ of the debris disks SEDs could be fitted with a single temperature blackbody $(T<45-85 \mathrm{~K})$ consistent with a ring-like configuration (Hillenbrand et al. 2008); detailed analysis of the excess spectra from $12-35 \mu \mathrm{m}$ of 44 of these stars showed that the inner radii of these cavities are $\sim 40 \mathrm{AU}$ for the disks with $70 \mu \mathrm{m}$ excess, and $\sim 10$ AU for the disks without $70 \mu \mathrm{m}$ excess (Carpenter et al. 2009). Inner cavities are also common around more massive stars: a Spitzer survey of 52 A-type and and late B-type stars known to have debris disks showed that the majority of the disks $(39 / 52)$ can be be fitted with a single-temperature blackbody with a median temperature of $190 \mathrm{~K}$, corresponding to a characteristic distance of $10 \mathrm{AU}$ (Morales et al. 2009). One caveat of the SED analysis is its degeneracy in the absence of spatially resolved observations and/or spectral features that are able to constrain the grain radius and composition. If the system is known to harbor planets, an additional constraint on the dust location can be obtained from dynamical simulations that study the effect of the planetary perturbations on the stability of the planetesimals' orbits, and that can identify the regions where the planetesimals could be stable and long-lived (Moro-Martín et al. 2007, 2010). To set tighter constraints on the planetesimal location, there is the need to obtain spatially resolved images and/or accurate photometric points from the mid-infrared to the submillimeter, so the inner and outer radius of the disk can be better determined. Observations with Herschel, JWST and ALMA will be very valuable for this purpose

\section{Prospects for the future: Herschel and beyond}

The on-going Hershel debris disks surveys DEBRIS and DUNES (Matthews et al. in preparation; Eiroa et al. in preparation - see proceeding in this volume) are designed to characterize debris disks around AFGKM stars at 70, 100 and $160 \mu \mathrm{m}$, with follow-up at 350, 450 and $500 \mu \mathrm{m}$; these observations are already doubling the Spitzer debris disk detection rate, increasing the number of spatially resolved disks, and allowing to characterize a new population of cold disks. ALMA's unprecedented high spatial resolution will be able to advance in the study of debris disk structure, and to test the models of planet-disk interactions; its long wavelength observations will allow to better constrain the disks outer radii. Debris disks surveys with JWST in the near to mid-infrared will 
allow to characterize the warm dust component, setting constrains on the frequency of planetesimal formation in the terrestrial planet region, and identifying stars with low debris dust contamination that may be good targets for terrestrial planet detection. Deep debris disks surveys with JWST and SPICA (the approval of the latter is pending) will be able to characterize debris disks around stars of a wide range of spectral types, ages and environment, studying the debris disk evolution and the dust production rate as a function of stellar age (the latter will help identify systems undergoing LHB-type of events, and to assess whether the dynamical evolution of the Solar system was particularly benign). SPICA/SAFARI (if approved) will also be able to study the dust composition by carrying out a spectral survey of debris disks and, for nearby disks, spectral imaging (that will allow to trace the variation in the dust mineral content as a function of disk radius, that can be compared to the compositional gradient in the Solar system - also to be studied by $S P I C A / S A F A R I)$. Regarding the Solar system, advancements need to be made in the study of its debris dust (e.g. with sample return missions to an asteroid and/or a comet, and dust detection experiments in the outer Solar system). Finally, there are programs to detect planets in debris disks stars using ground-based telescopes (e.g. Subaru/HiCIAO, Gemini/GPI and VLT/SPHERE) that will allow to study the planetdisk interaction. These are some of the future research lines in debris disk studies that will help us understand our Solar system in the context of the wide diversity of planetary systems.

\section{References}

Beichman, C. A., et al. 2005, ApJ, 626, 1061

Booth, M., et al. 2009, MNRAS, 399, 385

Bryden, G., et al. 2006, ApJ, 636, 1098

Carpenter, J. M., et al. 2009, ApJS, 181, 197

Dermott, S. F., et al. 1994, Nature, 369, 719

Dermott, S. F., et al. 2002, Asteroids, Comets, and Meteors: ACM 2002, 500, 319

Gautier, T., N. III, et al. 2007, ApJ, 667, 527

Gomes, R., Levison, H. F., Tsiganis, K., \& Morbidelli, A. 2005, Nature, 435, 466

Gurnett, D. A., Ansher, J. A., Kurth, W. S., \& Granroth, L. 1997, Geoph. R. Lett., 24, 3125

Grün, B. A. S., et al. 2001 in: Grün et al. (eds.), Interplanetary Dust (Springer), 295

Hillenbrand, L. A., et al. 2008, ApJ, 677, 630

Humes, D. 1980, J. Geophys. R., 85 (A/II), 5841

Jessberger, E. K. 2001, in: Grün et al. (eds.), Interplanetary Dust (Springer), 253

Jewitt, D. C. Luu, J. X. 2000, in: Mannings et al. (eds.), Protostars and Planets IV, 1201

Jura, M. 2006, $A p J, 653,613$

Landgraf, M., Liou, J.-C., Zook, H. A., \& Grün, E. 2002, AJ, 123, 2857

Lisse, C. M., Beichman, C. A., Bryden, G., \& Wyatt, M. C. 2007, ApJ, 658, 584

Lovis, C., Mayor, M., Pepe, F., Alibert, Y., Benz, W., et al. 2006, Nature, 441, 305

Meyer, M. R., et al. 2008, ApJL, 673, L181

Morales, F. Y., et al. 2009, ApJ, 699, 1067

Moro-Martín, A., et al. 2007, ApJ, 668, 1165

Moro-Martín, A., et al. 2010, ApJ, 717, 1123

Nesvorný, D., et al. 2010, ApJ, 713, 816

Reach, W. T., Morris, P., Boulanger, F., \& Okumura, K. 2003, Icarus, 164, 384

Strom, R. G., Malhotra, R., Ito, T., Yoshida, F., \& Kring, D. A. 2005, Science, 309, 1847

$\mathrm{Su}, \mathrm{K}$. Y. L., et al. 2006, ApJ, 653, 675

Sykes, M. V. \& Greenberg, R. 1986, Icarus, 65, 51

Trilling, D. E., et al. 2008, ApJ, 674, 1086

Wyatt, M. C., et al. 2007, ApJ, 658, 569 